\title{
GABA Withdrawal Modifies Network Activity in Cultured Hippocampal Neurons
}

\author{
H. Golan, ${ }^{1,2}$ K. Mikenberg, ${ }^{1}$ V. Greenberger, ${ }^{1}$ and M. Segal ${ }^{1 \dagger}$ \\ ${ }^{1}$ Department of Neurobiology, The Weizmann Institute of Science, Rehovot 76100; \\ ${ }^{2}$ Department of Virology and Development, Ben-Gurion University, Beer Sheva 84105; ISRAEL
}

\section{SUMMARY}

Dissociated hippocampal neurons, grown in culture for 2 to 3 weeks, tended to fire bursts of synaptic currents at fairly regular intervals, representing network activity. A brief exposure of cultured neurons to GABA caused a total suppression of the spontaneous network activity. Following a washout of GABA, the activity was no longer clustered in bursts and instead, the cells fired at a high rate tonic manner. The effect of removing GABA could be seen as long as 1 to 2 days after GABA withdrawal and is expressed as an increase in the number of active cells in a network, as well as in their firing rates. Such striking effects of GABA removal may underlie part of the GABA withdrawal syndrome seen elsewhere.

\section{KEYWORDS}

GABA withdrawal syndrome, multi-electrode array

\footnotetext{
${ }^{\dagger}$ Corresponding author:

Phone: 972 89342553, Fax: 97289344140

e-mail: jnsegal@weizmann.weizmann.ac.il

${ }^{\S}$ The initial experiments presented in this manuscript were conducted in collaboration with Dr. Simon Brailowsky during his visit to the Weizmann Institute in the Spring of 1997. The manuscript is dedicated to his memory.
}

\section{INTRODUCTION}

Exposure of neurons to GABA, the main inhibitory neurotransmitter in the brain, activates in these neurons a chloride conductance that causes a profound inhibition in the affected neurons for as long as GABA is present. Removal of chronically perfused GABA from intact brain tissue causes a characteristic increase in the excitability of the exposed neurons, to the extent that they may undergo severe epileptic seizures. This phenomenon, called the "GABA withdrawal syndrome (GWS)", has been studied extensively by Brailowsky ${ }^{\S}$ and colleagues (Garcia Ugalde et al., 1992 Silva-Barrat et al., 1992; 1995; Brailowsky \& Garcia 1999). GWS is assumed to be caused by a downregulation of the GABA receptor, such that GABA no longer inhibits cells, thus resulting in their inability to prevent seizures. GABA withdrawal syndrome has been assumed to activate mechanisms that are related to long-term memory, in that GWS can be expressed long after the removal of GABA from the tissue. GABA withdrawal syndrome has been associated with a number of morphological and biochemical changes in affected tissue (Arenda et al., 1994). The molecular mechanisms underlying GWS are largely unknown, partly because the syndrome has been produced only in vivo, where only a small volume of nervous tissue can be chronically exposed to GABA, and the analysis of its cellular and molecular mechanisms is rather limited. 
Tissue culture provides a simple in-vitro test system that is easily accessible to the biophysical and biochemical analysis of structure and function of central neurons, and where the GABAergic synapse has been studied extensively (Segal \& Barker, 1984a; 1984b). We explored the possibility of expressing GWS in cultured neurons and wish to report that even short exposures to GABA produce long-lasting changes in the activity patterns of small networks of central neurons maintained in culture.

\section{METHODS}

\section{Culture of hippocampal neurons}

Hippocampal cultures were prepared as described in Papa et al. (1995). Briefly, 19-day-old embryos were taken from anesthetized Wistar rats. The brains were removed and placed in ice-cold medium. The hippocampus was dissected out and mechanically dissociated by gentle trituration, using a Pasteur pipette. Dissociated cells $(800,000$

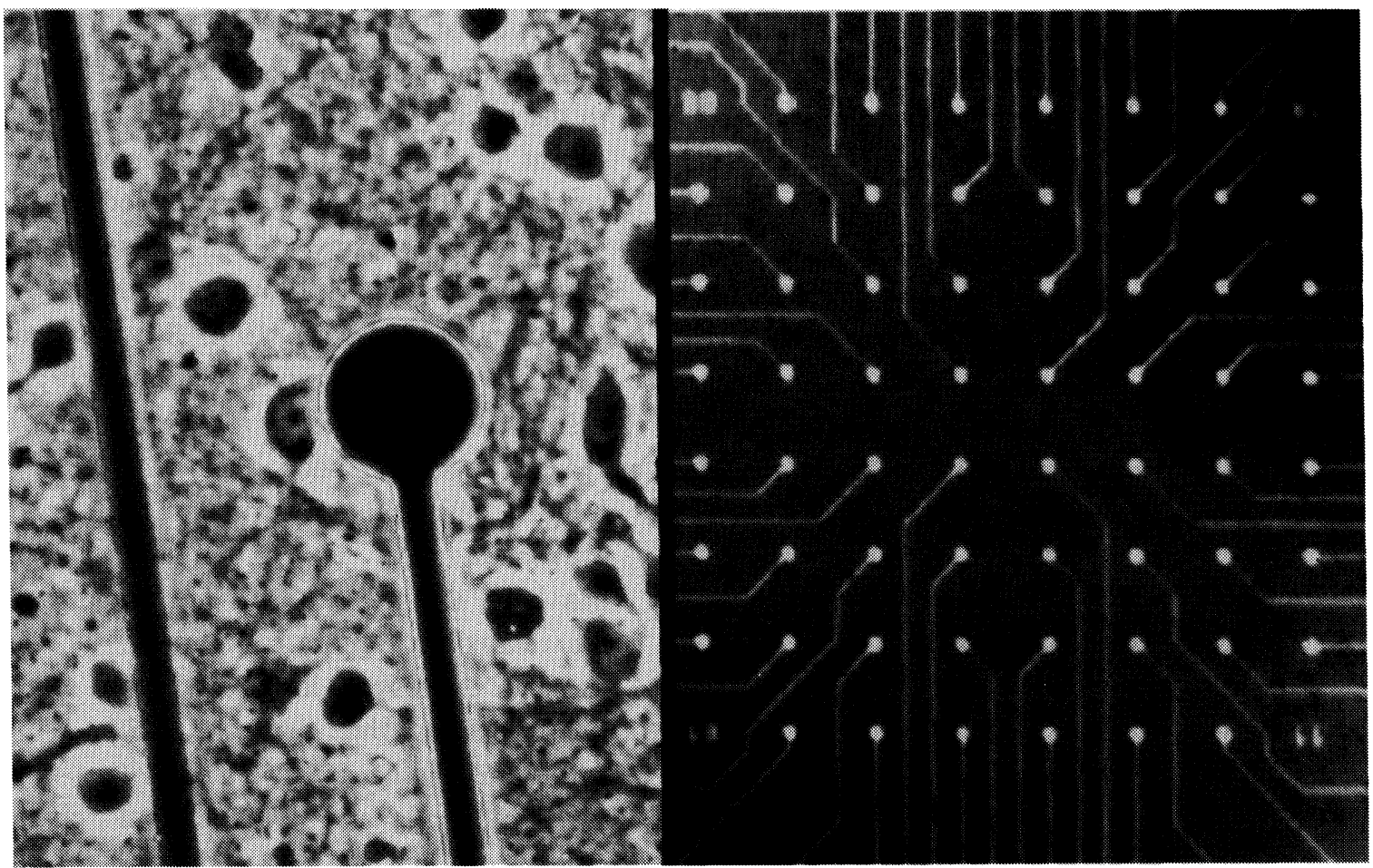

Fig. 1: The multi electrode recording system. Left, a low power view of the arrangement of the multielectrode surface. The distance between the electrode tips is $200 \mu \mathrm{m}$, and the size of each electrode tip is $20 \mu \mathrm{m}$. Right, a higher power view of a cell growing on an electrode tip, partially hidden by the electrode. The density of cell plating is such that an electrode is not likely to detect activity of more than one cell, although occasionally the activity of two cells is recorded simultaneously from an electrode. In such cases, the separation between them is quite clear (see below). 
cells $/ \mathrm{mL}$, as determined in a counting chamber) were plated onto $12-\mathrm{mm}$ glass coverslips or onto multi-electrode arrays (MEA, 60 electrodes, spaced $200 \mu \mathrm{m}$ apart, $20 \mu \mathrm{m}$ in diameter each, (Egert et al., 1988 and Fig. 1) that had been previously coated with $0.01 \%$ polyethylenimine in water. The plating medium was Eagle's MEM containing $10 \%$ heat-inactivated horse serum, $0.6 \%$ glucose, and gentamicin $(15 \mathrm{ug} / \mathrm{mL})$. Cells were incubated at $37^{\circ} \mathrm{C}$ in an atmosphere of $5 \%$ $\mathrm{CO}_{2}$. To prevent overgrowth of glial cells, the first change of medium, 5 days after plating, included $50 \mathrm{ug} / \mathrm{mL}$ uridine and $20 \mathrm{ug} / \mathrm{mL}$ deoxyuridine. Subsequent medium changes included MEM and $10 \%$ horse serum. Using these growth conditions, about $20 \%$ of the cells in culture were GABAergic (unpublished observations and Murphy et al,. 1998), and most of the medium-size cells (15 to $25 \mu \mathrm{m}$ ) were believed to be pyramidal cells. Within the first week in culture, the cells may migrate but are usually stable thereafter.

\section{Electrophysiological recording}

The effect of GABA application and withdrawal on neuronal activity was examined using intracellular, whole-cell patch recording and extracellular recording from a multi-electrode array.

\section{Whole cell recording}

The cultures were transferred to the recording chamber in an inverted Nikon microscope. The culture was perfused with a medium containing $130 \mathrm{mM} \mathrm{NaCl}, 5 \mathrm{mM} \mathrm{KCl}, 2 \mathrm{mM} \mathrm{CaCl}, 1 \mathrm{mM}$ $\mathrm{MgCl}_{2}$, and $30 \mathrm{mM}$ glucose. The $\mathrm{pH}$ was buffered to 7.4 with $25 \mathrm{mM}$ Hepes, and the osmolarity was adjusted with sucrose to $320 \mathrm{mOsm}$. Patch pipettes contained $140 \mathrm{mM} \mathrm{K}$-gluconate, $2 \mathrm{mM} \mathrm{Mg-ATP \text {, }}$ $0.2 \mathrm{mM}$ EGTA, $10 \mathrm{mM}$ Na-phosphocreatine, $5 \mathrm{mM} \mathrm{NaCl}$, and $0.3 \mathrm{mM}$ GTP. The $\mathrm{pH}$ was buffered to 7.2 with $10 \mathrm{mM}$ Hepes, and the osmolarity was adjusted to $290 \mathrm{mOsm}$. QX-314 $(5 \mathrm{mM})$ was added to block action potential discharges. Conventional whole-cell patch recording was conducted with a $1.5 \mathrm{~mm}$ OD glass pipette, having a $2 \mu \mathrm{m}$ tip and a resistance of 2 to $4 \mathrm{MOhm}$. Signals were amplified with an Axopatch 200 (Axon Instruments) amplifier. The cells were routinely held at $-60 \mathrm{mV}$, which is about their resting membrane potential. Drugs were perfused in the recording medium, and signals were stored on a PC computer, using Axon Instruments software.

\section{Multi channel recording}

Thirty minutes before the electrophysiological recording, the medium was changed to Hibernate A (Gibco Inc). Once the experimental procedure ended, the hibernate medium was replaced again by the bicarbonate-buffered medium, and between test sessions, the cultures were maintained in the $37^{\circ} \mathrm{C} / 5 \% \mathrm{CO}_{2}$ incubator. Spontaneous electrophysiological activity of selected neurons in the hippocampal culture were continuously monitored, using a 60-channel amplifier, 1060MEA (Multi channel system, Rutlingen, Germany). The culture chamber was equipped with a temperature controller to maintain a stable temperature at $37^{\circ} \mathrm{C}$. The culture density was low to obtain spike activity at each electrode from a single neuron. Neuronal activity was sampled over periods of $20 \mathrm{~min}$, using the Alpha-MAP acquisition program (Alpha Omega, Nazareth, Israel) and was analyzed off-line to determine the spike properties, the burst activity, and the dynamics of the network.

\section{Spike and burst analysis}

Analysis of the extracellular signals was made off-line; the mean value $(\mu)$ and standard deviation $(\sigma)$ of the background noise was estimated for each channel. Signals whose amplitude was greater than $\mu \pm 5 \sigma$ were identified as spikes. Each spike was defined by its amplitude and time of appearance for further analysis. The stability of firing frequency was verified by examining the firing along the sample duration. Only cells with stable firing 
rates were analyzed. Action potentials of the same shape and size could be recorded over periods up to a week. Lowpass digital filtering was used to define bursts of spikes. The analysis included the evaluation of interspike intervals, interburst intervals, and averaged amplitudes. In preliminary experiments, we examined cross-correlations between active channels.

\section{RESULTS}

\section{Single cell recordings}

At the age of 2 to $3 \mathrm{wk}$ in culture, patchclamped neurons expressed spontaneous rhythmic bursting activity (Fig. 2) at the rate of one burst every 2 to $10 \mathrm{sec}$. Each burst consisted of many EPSCs and IPSCs discharged simultaneously for about $500 \mathrm{msec}$. The IPSCs could be easily distinguished from the EPSCs by their reversal potential at about $-50 \mathrm{mV}$. Perfusion of $50 \mu \mathrm{M}$ GABA into the culture produced an immediate and long-lasting cessation of spontaneous synaptic currents and an inward current that was associated with a marked increase in membrane conductance. Within a minute of the onset of GABA perfusion, the inward current sagged but then remained constant thereafter for the entire duration of GABA presence in the dish. Likewise, the conductance change caused by GABA was not reduced, indicating that at least for $10 \mathrm{~min}$ of GABA presence, no additional desensitization of the GABA receptor occurred beyond a possible fast one. In all 7 cells tested, each in a different culture dish, washout of GABA from the recording chamber, after either 1 or $10 \mathrm{~min}$ of exposure to the drug, resulted in a rapid return of the membrane current and conductance to predrug levels. Recovery was followed by a dramatic increase in EPSC and IPSC discharges in a tonic manner (Fig. 2). The high-frequency, continuous non-bursting behavior lasted about 10 to $20 \mathrm{~min}$ and was eventually recovered to normal bursting activity over the subsequent 30 min. To test if the enhanced activity was a result of the continuous lack of rhythmic activity caused by GABA, we exposed three cells to $1 \mu \mathrm{M}$ tetrodotoxin (TTX), which also caused cessation of the action potential and spontaneous bursting. Following the removal of TTX, bursting activity was restored, and no long-lasting effects of TTX were recorded (data not shown), in sharp contrast to the effects of GABA withdrawal.

\section{Multi-channel recording}

To further characterize this unique short term effect of GABA withdrawal and extend it to hours and days after treatment, we resorted to a similar treatment of hippocampal cultures plated on MEA (Fig 3). Recording the extracellular activity from the culture enabled us to monitor the behavior of many neurons in a non-invasive manner and to follow changes in their intrinsic firing properties and their interconnections over periods of up to 2 wk in culture (Maeda et al. 1995). At the age of 1 to $2 \mathrm{wk}$, activity was frequently detected in 5 to 6 of the 60 electrodes. Hippocampal neurons in a 9-dayold culture expressed moderate levels of activity, averaging $0.6 \mathrm{~Hz}$. Most cells discharged highfrequency bursts, interspersed with long periods of quiescence, with a preference for a 2 to $8 \mathrm{sec}$ interburst interval (Fig. 3), in much the same way as that seen with the intracellular recording shown in Fig. 2 above. Exposure to GABA $(20 \mu \mathrm{M})$ for 3 to 5 min totally suppressed action potential discharges in all of the recorded cells $(n=25)$. Fast washout of GABA resulted in most of the cells in a high frequency of spike firing and complete disappearance of slow burst-firing pattern. An example of the activity of 4 different cells before the GABA application, within $10 \mathrm{~min}$, and $24 \mathrm{~h}$ after the GABA washout, is presented in Fig. 4. As seen in this example, all cells showed a 2- to 4-fold increase in spike frequency within the first 10 min after GABA washout. The same trend 


\section{Before}
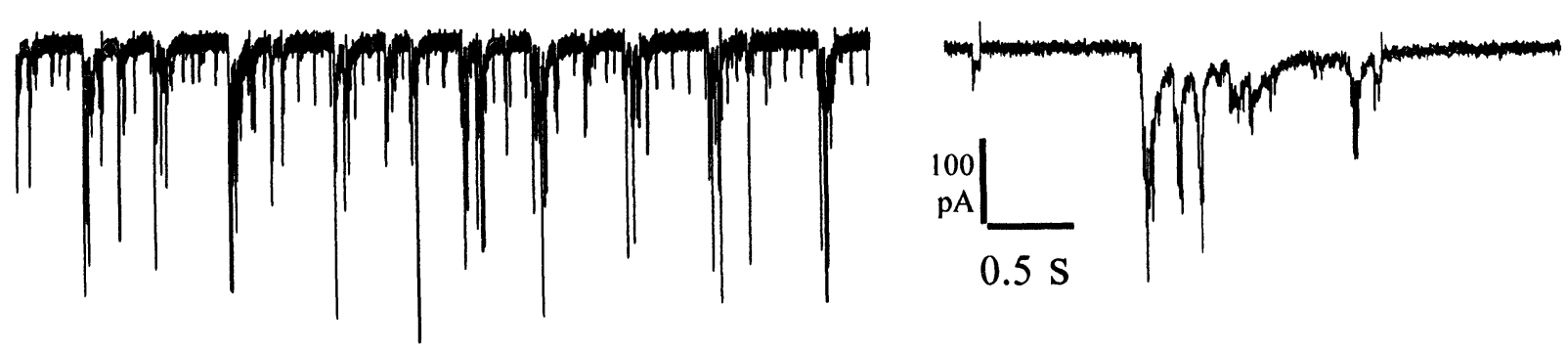

\section{GABA}
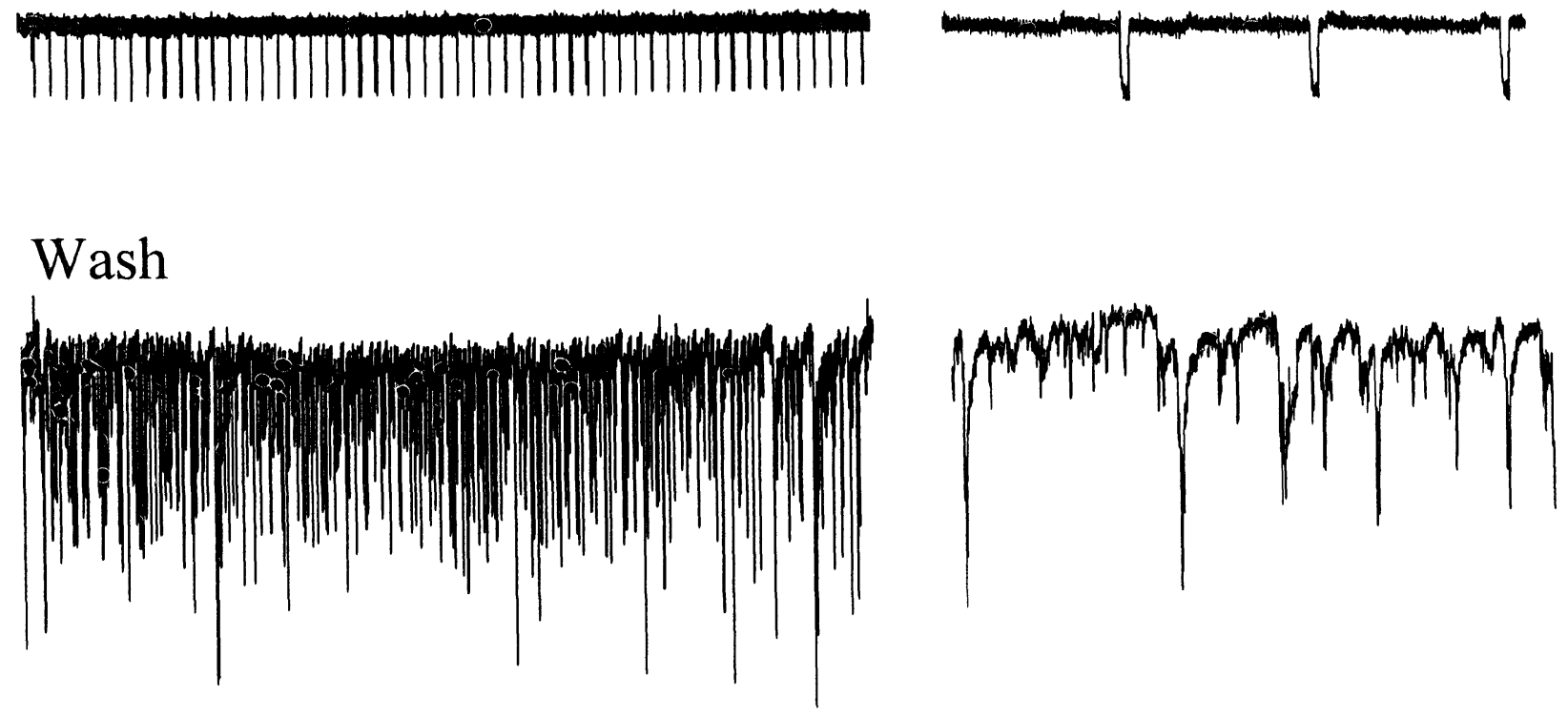

Fig. 2: Spontaneous activity recorded from a patch-clamped hippocampal neuron growing in culture for 3 weeks. Cell clamped at $-60 \mathrm{mV}$, and negative voltage commands are applied through the membrane to estimate input conductance of the cell. Left, 1 minute record, right an expanded record to show a typical burst of synaptic currents, and a preceding current response to voltage command. Current scale same for all, time scale only for the right, expanded records. During exposure to $50 \mu \mathrm{M}$ GABA, middle trace, there is complete suppression of synaptic current, and a marked increase in membrane conductance. Bottom, following washout of GABA, EPSCs lost their bursting properties, and a continuous barrage of synaptic currents is seen. 


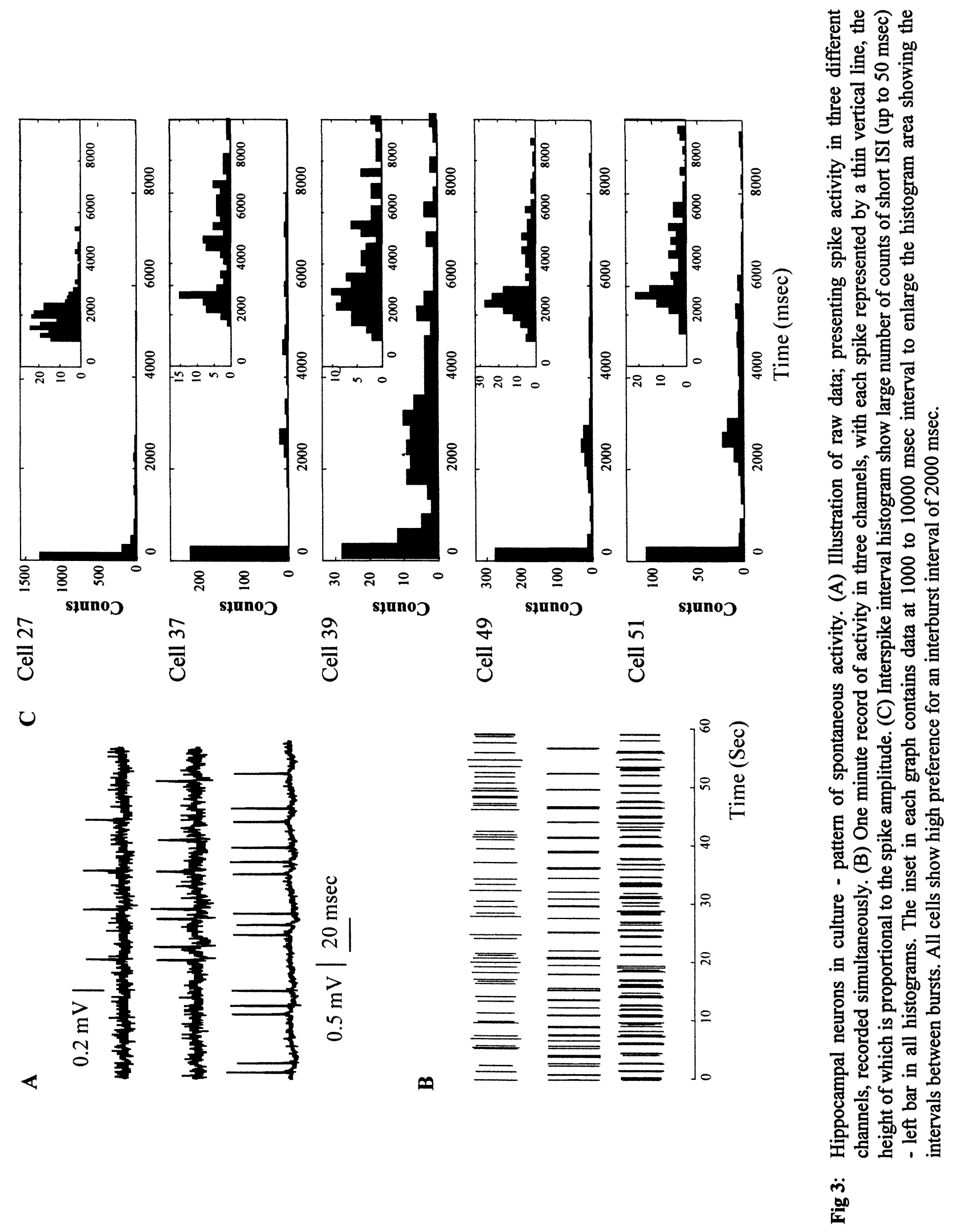




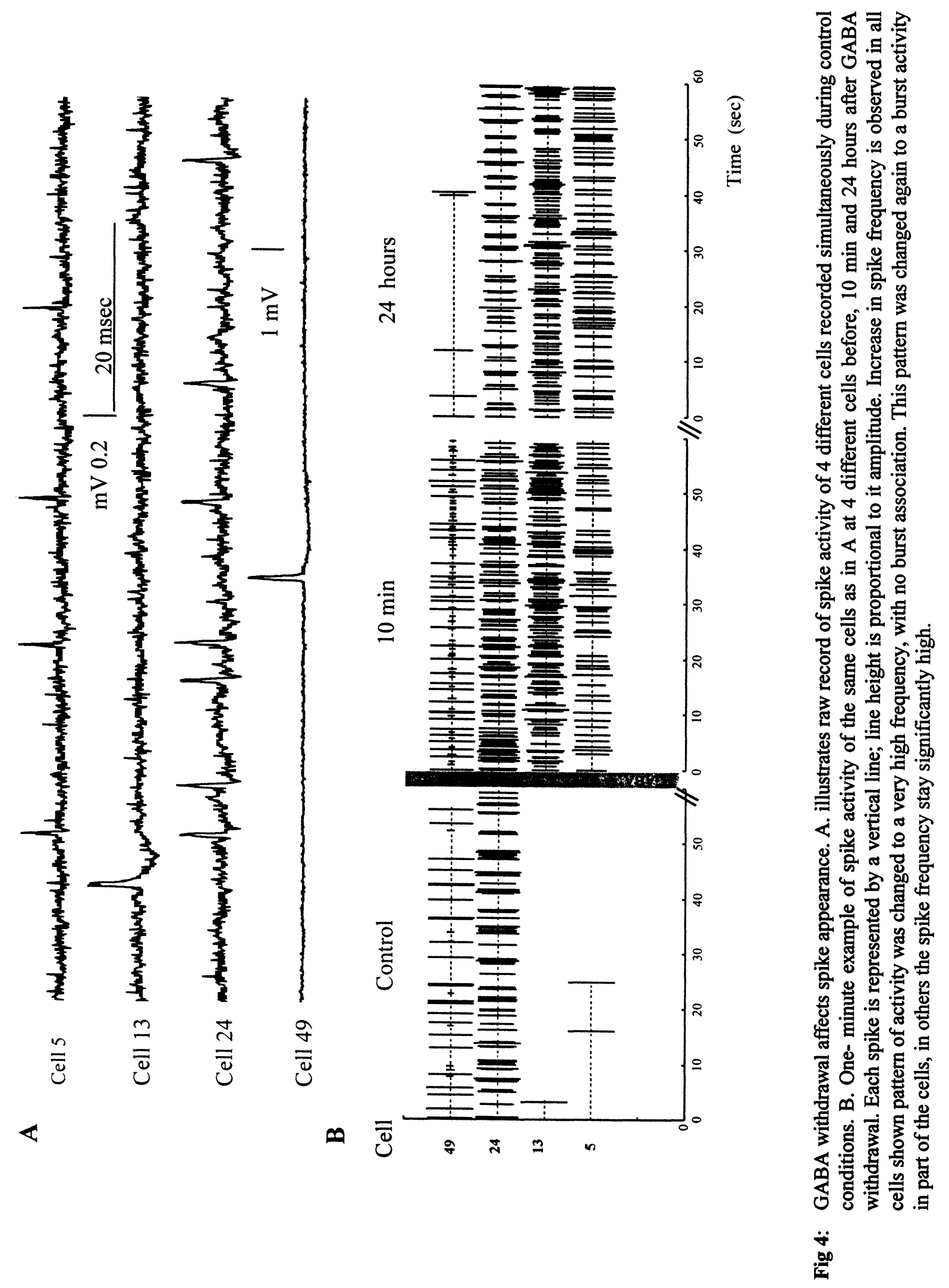


was seen in the next $10 \mathrm{~min}$ (data not shown). All cells fired in a bursting mode a day later. The bursts were now fired at higher frequencies, however. High rates of activity and a change in firing frequency are also illustrated in Fig. 5 by the interspike histogram (ISI). Most cells tested (8/12) expressed a clear increase in firing frequency, in addition to the change in the pattern of activity. Table 1 presents the change in spike frequency in 10 cells at different time points after GABA withdrawal. Although the high rate of spike firing that was seen immediately after the GABA washout gradually declined, yet $24 \mathrm{~h}$ after exposure to GABA, the average spike rate of the network was higher than that under control conditions.

\section{TABLE 1}

GABA withdrawal effect on spike frequency, short and long term effects ${ }^{\ddagger}$

\begin{tabular}{|c|c|c|c|c|}
\hline Cell \# & Control & $10 \mathrm{~min}$ & $20 \mathrm{~min}$ & $24 \mathrm{~h}$ \\
\hline 32 & 0.297 & 1.3 & n.a. & 1.8 \\
\hline 52 & 0.828 & n.a. & n.a. & 2.5 \\
\hline 13 & 0.013 & 0.5 & n.a. & 0.58 \\
\hline 18 & 0.29 & 1.3 & n.a. & 0.2 \\
\hline 29 & 0.58 & 4.1 & n.a. & 0.83 \\
\hline 31 & 1.1 & 0.6 & n.a. & 0.13 \\
\hline 5 & 0.225 & 0.74 & 0.26 & 1.41 \\
\hline 13 & 0.08 & 2.32 & 2.25 & 2.16 \\
\hline 24 & 2.1 & 1.16 & 0.4 & 3.75 \\
\hline 49 & 0.58 & 1.2 & 1.16 & 0.11 \\
\hline Mean & 0.609 & 1.468 & 1.017 & 1.347 \\
\hline SEM & 0.062 & 0.124 & 0.227 & 0.120 \\
\hline N & 10 & 9 & 4 & 10 \\
\hline P & & 0.042 & & 0.025 \\
\hline
\end{tabular}

${ }^{\ddagger}$ Frequencies in $\mathrm{Hz} ; \mathrm{P}$ values for the one-tailed, paired t-test; $n . a=$ data not available

\section{Long-term effects of GABA withdrawal}

Table 1 shows that in all experiments, $24 \mathrm{~h}$ after a brief exposure to GABA, an increase in spike frequency still occurred, and a remarkable increase in the number of active cells was observed. Twenty-four hours after GABA withdrawal, more than a 2-fold increase in the number of active cells was observed (from 12 to 27 cells), whereas in a control culture of similar age that was exposed only to a change of medium, the number of cells did not change much (from 31 to 36 cells) during the 24-h incubation period. The pronounced increase in the number of active cells, in addition to the increase in spike frequency, resulted in an overall increase in the activity of the whole culture, as expressed by the total number of spikes/sec per culture. Although the cells that were sampled are only a small fraction of the total cell population in the culture, their activity represents the network activity. An average of 3.25 spikes/sec per culture was sampled before GABA and 8.55 spikes/sec $24 \mathrm{~h}$ later.

Furthermore, the cells not expressing burst activity, as well as those that did, developed a clear burst activity during the hour after GABA withdrawal, as shown in Fig. 5. This burst activity was still observed $24 \mathrm{~h}$ and $96 \mathrm{~h}$ later. A clear example of this tendency can be seen in cells 32 and 52, shown in Fig. 6. Cell 58 from the same culture fired at less then one spike per min before exposure to GABA (not shown because of the small number of spikes), but then switched to burst activity over the next $24 \mathrm{~h}$.

\section{DISCUSSION}

The experiments presented here demonstrate that exposure to GABA can alter the properties of a small hippocampal network that is maintained in culture. This small network, probably consisting of several hundreds of interconnected neurons, 

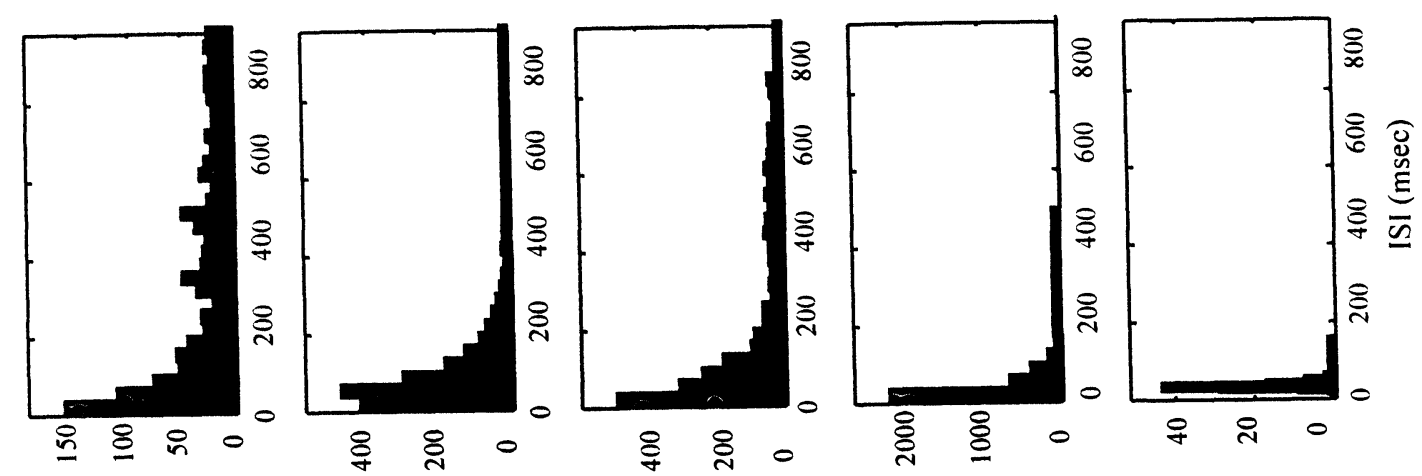

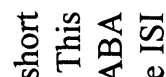

ᄋ

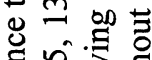

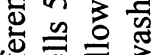

巴)

至

돈인

至嵌离

등 응

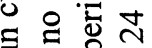

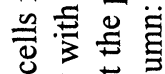

范

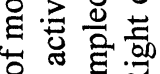

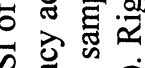

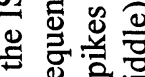

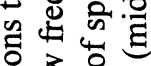

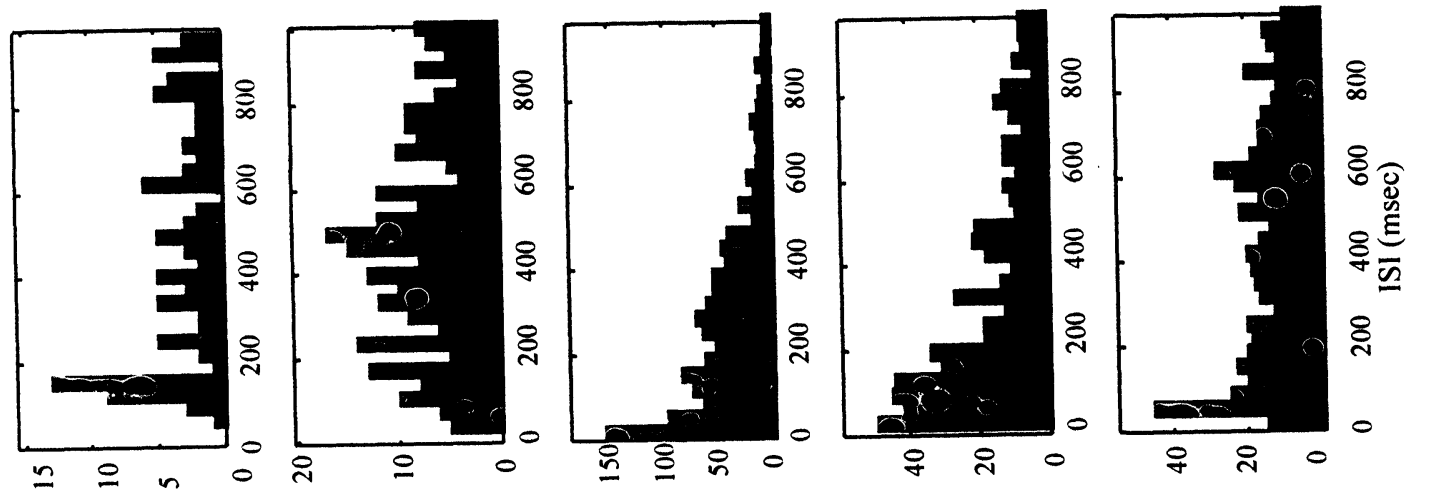

흐를 틀

ర하유

은 点

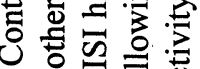

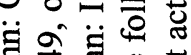

킁혀웛

幽芯芩

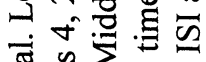

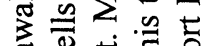

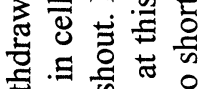

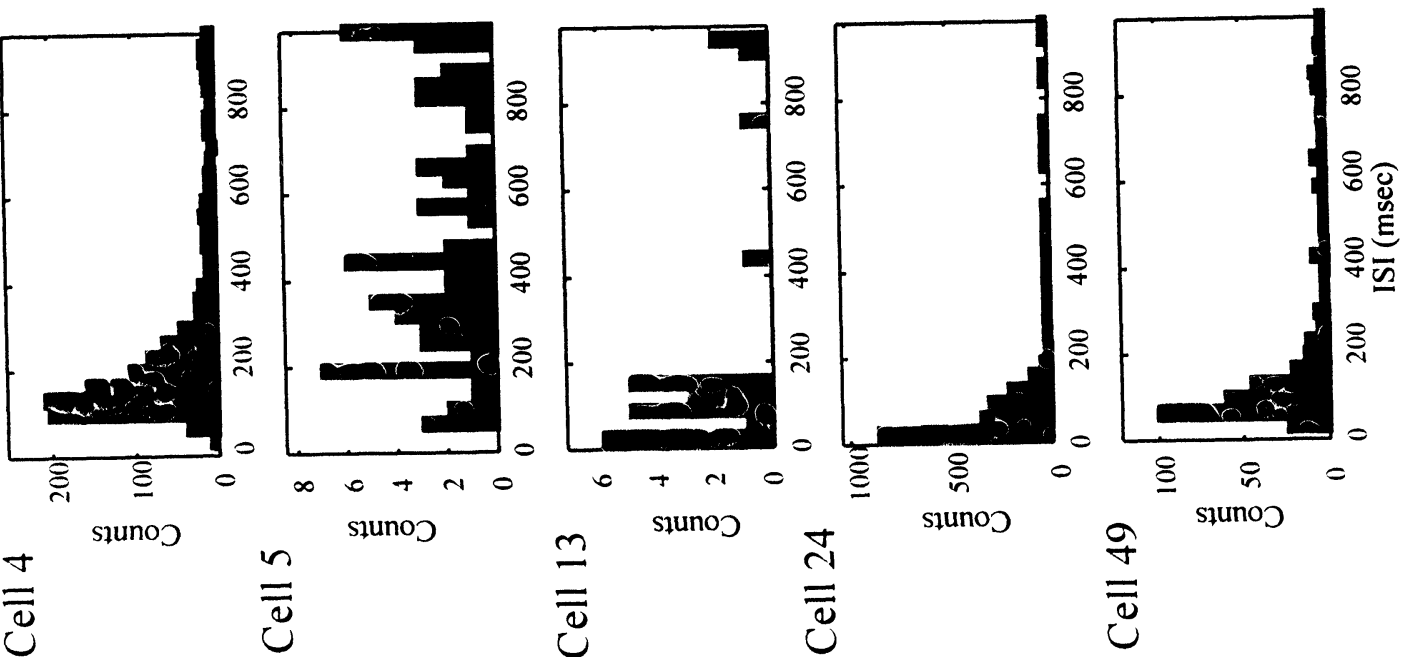

ฮี ญี $\varangle \mathbb{S}_{0}$ ๓ $\approx$

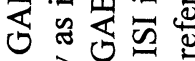
入 己

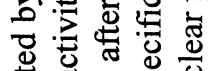

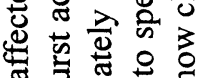
幽营 웅 흘 ญ

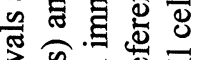
总总芯 으은영 옹 도유 总过芯 \% 舟 


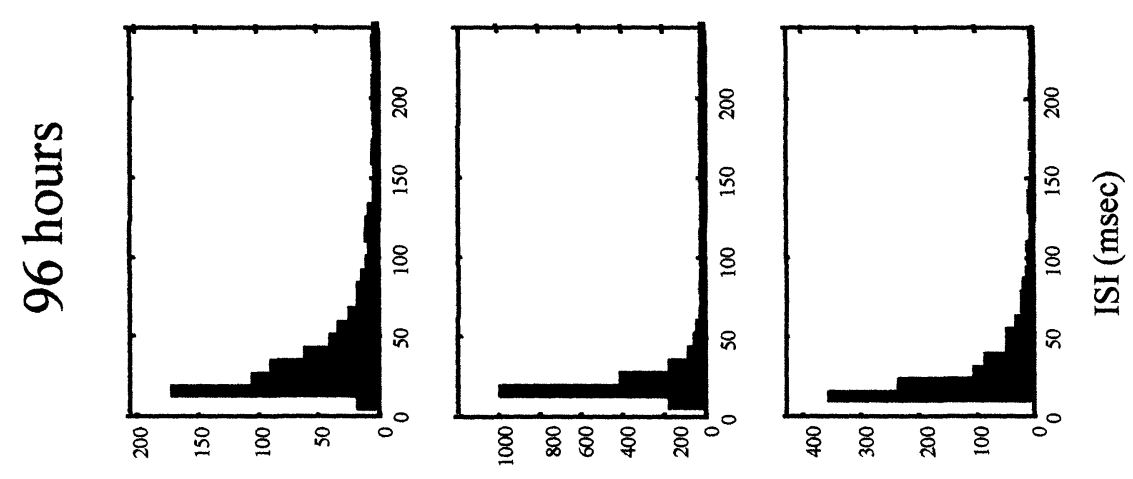

꼻 ๓

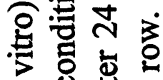
. 워ㅇㅝㅠ. 究혈 원영 पू 娄 है

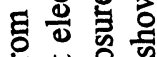
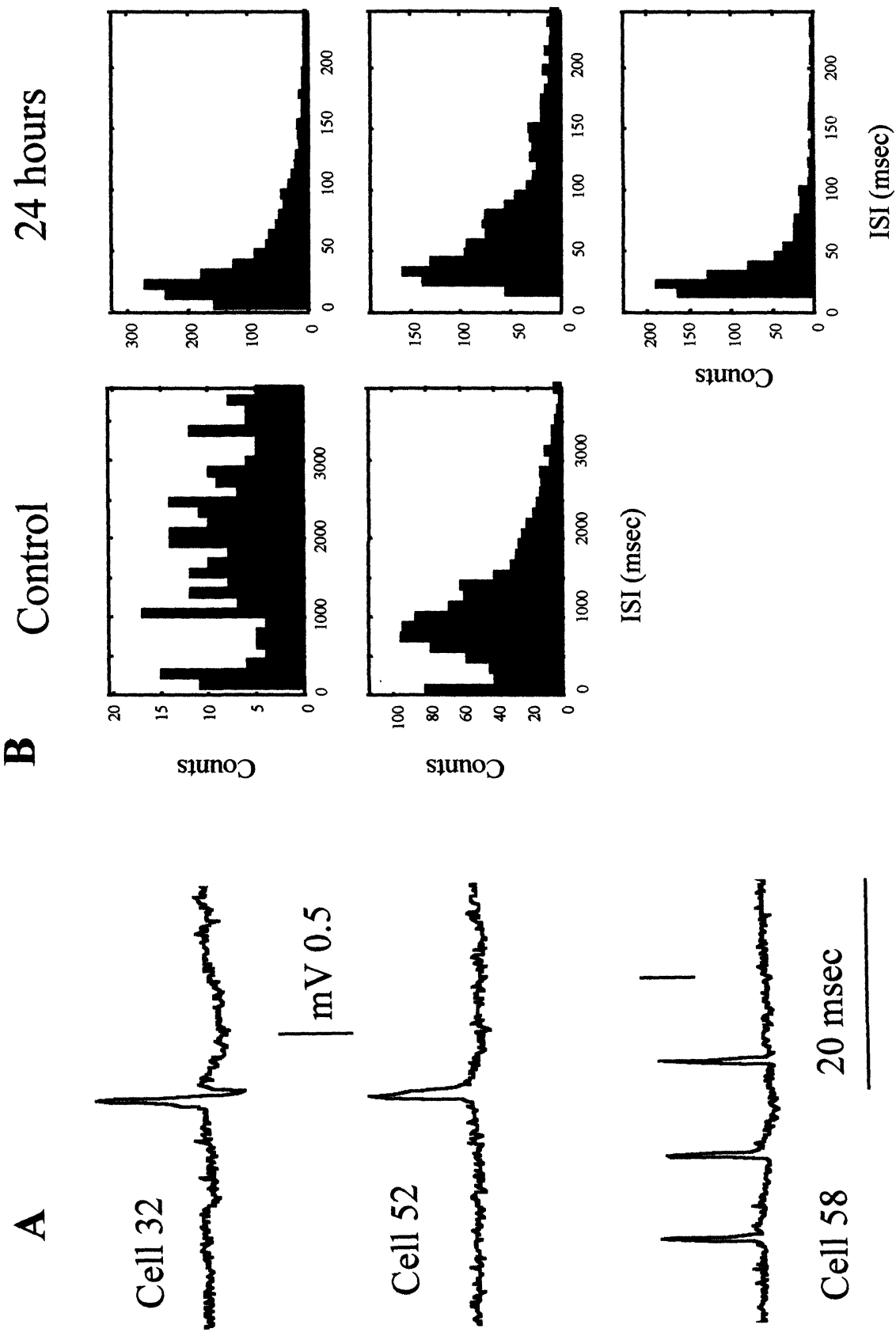

薦家.

边密

过

ङ

数范

造

妾高

की 造总

零致

월

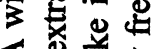

乐范旁言

ษ。.

递

要焉 存定宫要管

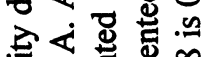

<

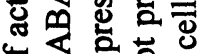

论 记苛 E $\sum_{3}$ 요유. $\cong \%$ 密

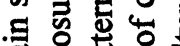

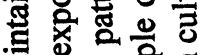
의 울

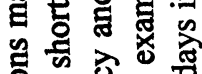
5 的

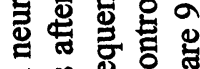

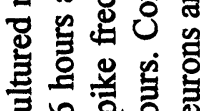
寻边总总 盛 
produced coordinated activity, expressed as bursts of synaptic currents appearing at fairly regular intervals. This network activity was suppressed by GABA. When the drug was washed out, however, the regular bursting activity was replaced by a highfrequency tonic activity. When recorded extracellularly with the multielectrode system, the enhanced synaptic activity was expressed as an increase in the total firing rate of the recorded cells and in the recruitment of "dormant" cells to the network. These effects lasted for at least 1 to $2 \mathrm{~d}$ after the washout of GABA.

Whereas the phenomenon reported here is not identical to the GWS studied in-vivo, where the neural tissue is chronically exposed to GABA, the results of GABA withdrawal reported here are qualitatively similar in that a dramatic increase in the tone of the network activity was seen for at least several days. Acute exposure to GABA is not expected to result in a major effect on the properties of the GABA receptor or on its associated chloride channel and indeed, continuous recording of membrane currents during a 1 to 10 min exposure to GABA did not reveal any change in conductance or any reduction beyond the initial sag in the inward current produced by GABA. The initial sag is likely to be caused by a redistribution of chloride ions across the membrane (Segal \& Barker, 1984). Thus, it is not likely that a change in GABA receptor properties underlies the change in network activity observed here. Nor is it likely that the cessation of activity per se, caused by exposure to GABA, underlies the increase in subsequent activity, as this effect was not mimicked by exposure of the culture to tetrodotoxin, a drug producing a similar suppression of activity but without the withdrawal effect. Thus, our in vitro GWS is not likely to represent a sheer overshoot of cells following their suppression but rather to result from a transient reduction in the efficacy of local interneurons in regulating the burst firing of pyramidal neurons. Such a reduction in efficacy may have long-lasting consequences, as seen here and elsewhere (Brailowsky et al., 1995), and may produce a long-lasting facilitation of network activity.
The site of action that is most sensitive to GABA exposure is not yet known.

Young interneurons in the developing brain have been shown to excite rather than to inhibit follower neurons because in young cells, the chloride reversal potential is more depolarized than the firing threshold (Cherubini et al., 1991). Such is not the case with the cells studied here. Although the use of a patch pipette did not allow the exact determination of the chloride reversal potential in the undisturbed neuron, the results of other studies using cell-attached patch recording (Murphy et. al., 1998) indicate that for cells at an age similar to that used in our experiments, from 1 to 3 weeks of culture, GABA is inhibitory. The ability of GABA to evoke an inward current in our cells, as recorded with the patch pipette, entails the reversal potential of chloride, which is $10 \mathrm{mV}$ below our standard holding potential $(-60 \mathrm{mV})$.

Because of their strategic locations and functions, GABAergic interneurons are the targets for extensive modulation in central circuits. Such interneurons are innervated by an array of extrinsic modulatory systems, including serotonin-, acetylcholine-, and noradrenaline-containing terminals (Freund \& Buzsaki, 1996), and by local excitatory and inhibitory collaterals. In the hippocampus, where local interneuronal circuits have been studied extensively, subsets of interneurons contain selective calcium-binding proteins and neuropeptides. Interneurons regulate network activity in the hippocampus, both via the activation of short-lasting GABA-A receptors, and longer-lasting GABA-B receptors. Thus, the ability to regulate network activity by interacting with a minimal number of GABAergic neurons is much more efficient than an interaction with the more abundant pyramidal neurons (Yanovsky et al., 1997). Our present results indicate that even a short exposure of a network to GABA can have long-lasting consequences to the network activity. Thus, intensive GABAergic activity, caused by drug-mediated release of GABA from terminals, may, upon its termination, cause the system to undergo a period of extensive excitation. This 
phenomenon may have long-term consequences for the ability of the network to react to excitatory afferents and may produce a withdrawal syndrome, expressed at different levels of neuronal activity.

\section{REFERENCES}

Araneda S, Silva-Barrat C, Menini C, Naquet R. High expression of noradrenaline choline acetyltransferase and glial fibrillary acidic protein in the epileptic focus consecutive to GABA withdrawal. An immunocytochemical study. Brain Res. 1994; 655: 135-146.

Brailowsky S, Garcia O. Ethanol, GABA and epilepsy. Arch Med Red 1999; 30: 3-9.

Brailowsky S, Montiel T, Meneses S, DiScala G. Effects of GABAB receptor antagonists on two models of focal epileptogenesis. Brain Res 1995; 702: 126-32.

Cherubini E, Gaiarsa JL, Ben-Ari Y. GABA: an excitatory transmitter in early postnatal life. Trends Neurosci. 1991; 14: 515-519.

Egert U, Schlosshauer B, Fennrich S, Nisch W, Fejtl M, Knott T, et al. A novel organotypic long-term culture of the rat hippocampus on substrate-integrated multi-electrode arrays. Brain Res Protocols 1988; 2: 229-242.

Freund TF, Buzsaki G. Interneurons of the hippocampus. Hippocampus 1966; 6: 347-370.

Garcia-Ugalde G, Galarraga E, Bargas J, Brailowsky S. Hyperexcitability of hippocampal CA1 region in brain slices after GABA withdrawal. Neurosci Lett 1992; 147: 229-232.
Maeda E, Robinson HP, A. Kawana. The mechanisms of generation and propagation of synchronized bursting in developing networks of cortical neurons. J Neurosci 1995; 15: 68346845.

Murphy DD, Cole NB, Greenberger V, Segal M. Estradiol increases dendritic spine density by reducing GABA neurotransmission in hippocampal neurons. J Neurosci 1998; 18: 2550-2559.

Papa M, Bundman MC, Greenberger V, Segal M. Morphological analysis of the development of dendritic spines in primary cultures of hippocampal neurons. J Neurosci 1995; 15: 1-11.

Segal M, Barker JL. Rat hippocampal neurons in culture: Properties of GABA-activated $\mathrm{Cl}$ ion conductance. J Neurophysiol 1984a; 51: 500-515.

Segal M, Barker JL. Rat hippocampal neurons in culture: voltage clamp analysis of inhibitory synaptic connections. J Neurophysiol 1984b; 52: 469-487.

Silva-Barrat C, Araneda S, Menini C, Champagnat $\mathrm{J}$, Naquet R. Burst generation in neocortical neurons after GABA withdrawal in the rat. $\mathrm{J}$ Neurophysiol 1992; 67: 715-727.

Silva-Barrat C, Champagnat J. A potassium current controls burst termination in rat neocortical neurons after GABA withdrawal. Neurosci Lett 1995; 189: 105-108.

Yanovsky Y, Sergeeva OA, Freund TF, Haas HL. Activation of interneurons at the stratum oriens/alveus border suppresses excitatory transmission to apical dendrites in the CA1 area of the mouse hippocampus. Neuroscience 1997; 77: 87-96. 

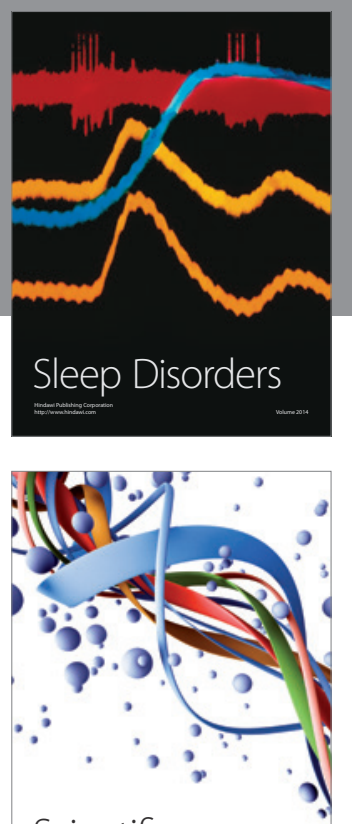

Scientifica
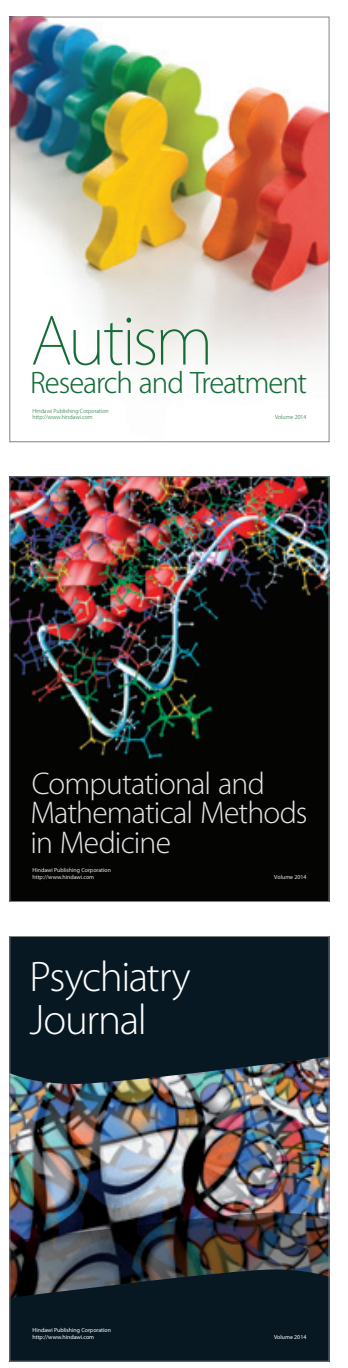
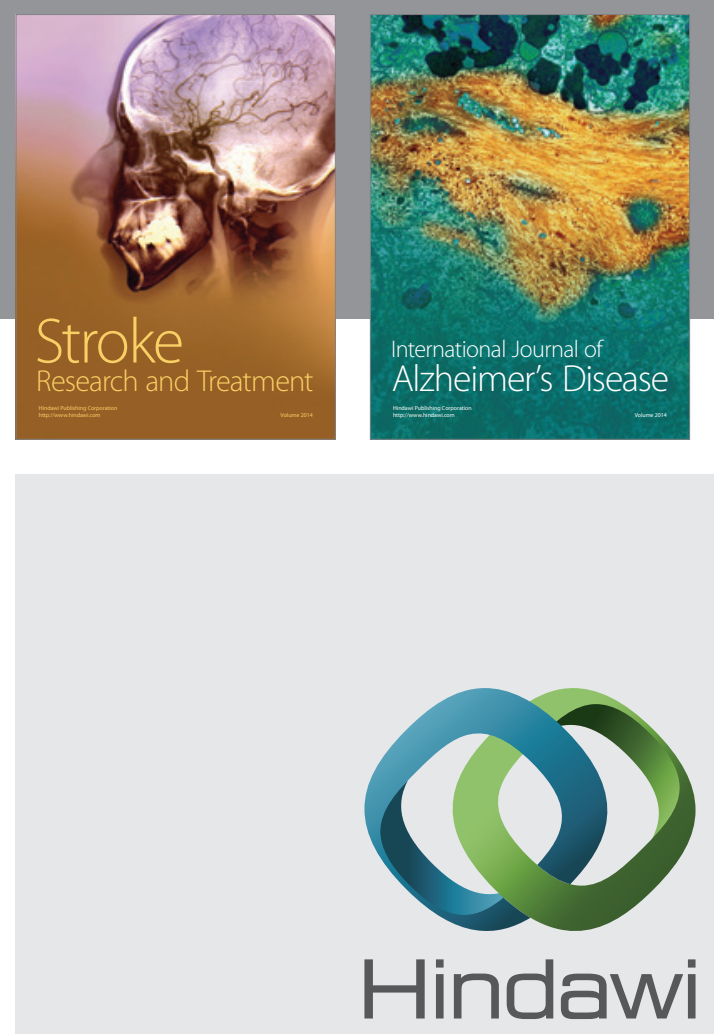

Submit your manuscripts at

http://www.hindawi.com
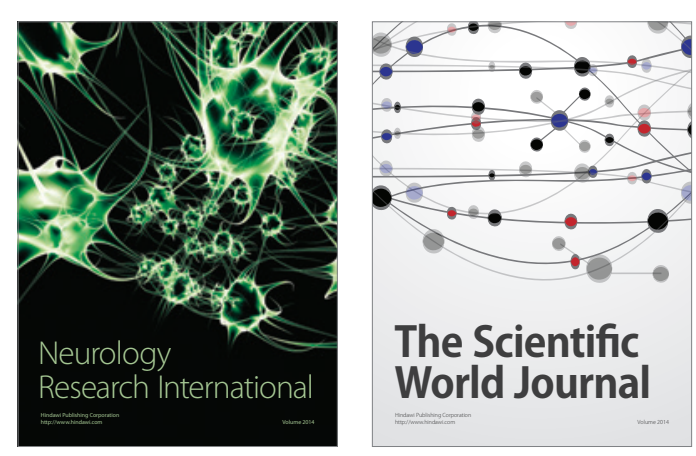

The Scientific World Journal

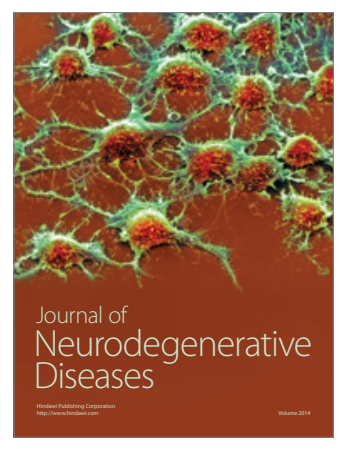

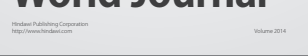

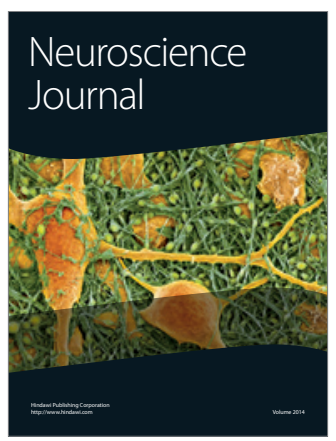

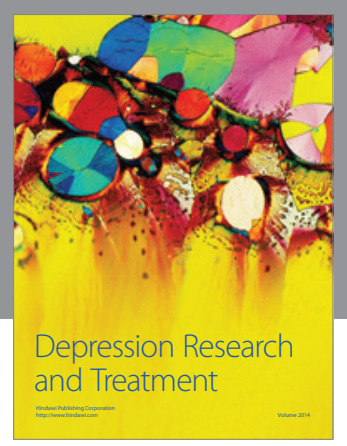
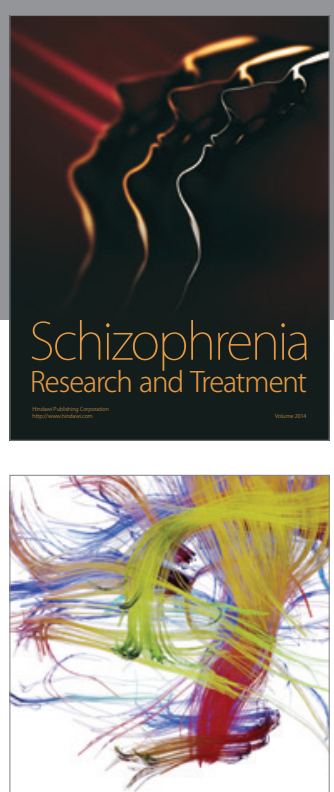

Brain Science

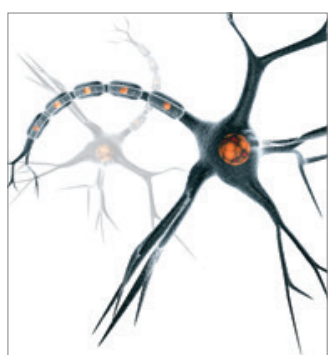

Neural Plasticity
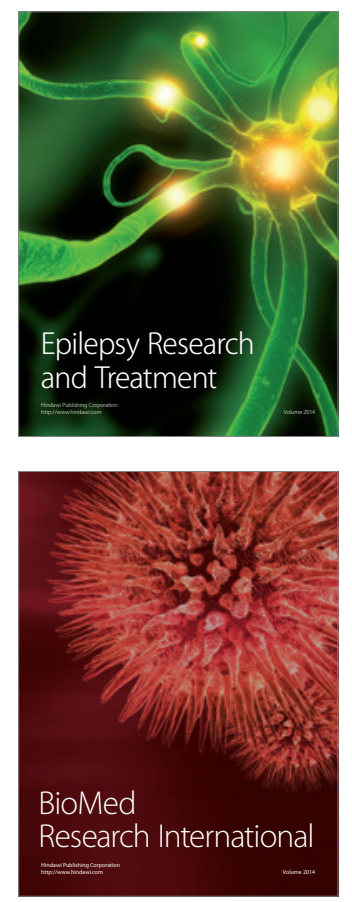

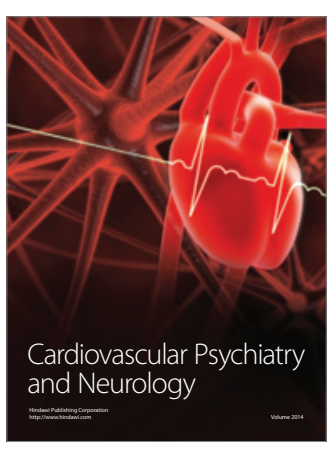

Parkinson's

Disease
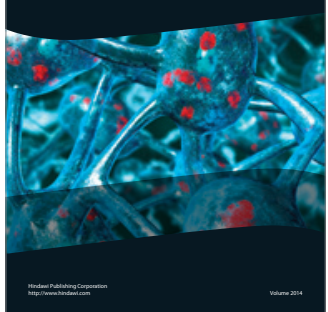